\title{
The Clinical Strategy to Overcome EGFR-TKI Acquired Resistance for NSCLC Patient
}

\author{
Rui Han', Chong Zhang' ${ }^{2}$ Li Li', Yong He ${ }^{1 *}$ \\ ${ }^{1}$ Department of Respiratory Disease, Daping Hospital, Third Military Medical University \\ ${ }^{2}$ Chongqing Key Laboratory of Molecular Oncology and Epigenetics, First Affiliated Hospital of Chongqing Medical University
}

*Corresponding author: Yong He, Department of Respiratory Disease, Daping Hospital, Third Military Medical University; E-mail: heyong@dphospital.tmmu.edu.cn

\begin{abstract}
Epidermal growth factor receptor tyrosine kinase inhibitors (EGFR-TKIs) are current treatments for advanced non-small cell lung cancer (NSCLC) harboring activating EGFR gene mutations. Although studies show an increased progression free survival (PFS) with use of EGFR TKIs in the first-line setting, most patients will develop resistance to therapy after the first about 10 months. Undoubtedly it is critical to choose an optimal clinical strategy for patients with EGFR sensitive mutation undergoing EGFR-TKI resistance. The second biopsy should be applied under condition permission to verify specific resistance mechanism. Here we discussed the mechanism of drug resistance and the choice of therapeutic regimen, also compared the superior and inferior of each treatment plan, which proposed a novel perspective for NSCLC target therapy.
\end{abstract}

Keywords: GFR-TKI; Resistance; T790M; Lung cancer
Received Date: January 20, 2016

Accepted Date: May 5, 2016

Published Date: May 11, 2016

Citation: He, Y., et al. The Clincal Strategy to Overcome EGFR-TKI Acquired Resistance for NSCLC Patient. (2016) Intl J Cancer Oncol 3(1): 1- 4.

DOI: $10.15436 / 2377-0902.16 .027$

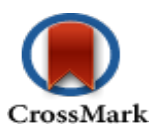

\section{Introduction}

\section{EGFR-TKI resistance and mechanisms}

Epidermal growth factor receptor tyrosine kinase inhibitor ( EGFR-TKI) has become a milestone in lung cancer treatment field in recent years ${ }^{[1]}$, EGFR sensitive mutations of advanced non-small cell lung cancer (NSCLC) treated with EGFR-TKI, progression-free survival (PFS) and objective response rate (ORR) were significantly improve ${ }^{[2]}$. However, EGFR-TKI resistance becomes the limiting of EGFR - TKI clinical application. Even the patients who EGFR-TKI initial treatment effectively, most of them will inevitably occur in drug resistance in 10 months $^{[3,4]}$.

EGFR-TKI resistance including primary resistance and acquired resistance. Primary drug resistance which also called baseline resistance or refractory to a drug is point to despite the presence of treatment benefit related mutations, but there are no clinical benefits after using TKI, and the disease progression is still occurred. Approximately 7-13\% NSCLC patients with sensitive mutations acquired no benefits during first-line TKI treatment. Patients with aberrant activation of the driving genes such as KRAS are expected to represent no response to EGFR-TKI treatment and this circumstance is insensitive to drugs which are not classified into primary drug resistance. Secondary resistance which also called acquired resistance refers to after the acceptance of EGFR-TKI treatment ,clinical benefit (tumor response, progress postponement, symptom relief) and subsequent disease progression will occur in all the NSCLC patients with EGFR gene sensitive mutation and effective EGFR-TKI initial therapy ${ }^{[5,6]}$.

The mechanisms of acquired resistance provided for two categories: Oncogene dependence and non-oncogene dependence. Epithelial mesenchymal transition of tumor cells and transformation of NSCLC to small cell lung cancer are the main contributors to the non oncogene dependence. Whereas the factors of oncogene dependence are highly related with genes second mutations including EGFR, HER, BRAF and other genes abnormal activation or amplification such as C-MET, PI3K, IGF1-R ${ }^{[6-8]}$. Various therapies after secondary resistance should be performed according to different reasons. For example, EP chemotherapy can be used 
for SCLC transformation; associated pathway inhibitors combined with TKI treatment can be applied for genes abnormal activation and amplification. Currently, all the MET, HGF, HER, IGF1R, AXL, MEK, AKT, PI3K, HSP90 inhibitors are in the development of clinical research, of which the outcomes are worth looking forward to ${ }^{[9,10]}$.

T790M, the second mutation of EGFR gene, is considered to be the main causes of primary EGFR-TKI resistance, accompany with secondary resistance of EGFR-TKI ${ }^{[1,12]}$. Studies have shown that T790M changes the spatial configuration of EGFR tyrosine kinase domain, which hinders the binding of TKI to EGFR receptor and weakens the effective competitive ability between TKI and ATP, eventually leading to resistance. Clinically, T790M mutations are commonly associated with sensitive mutations ${ }^{[4,5]}$ In recent years, according to newly reports about T790M mutation from China, Japan and United States, using various detection methods including arms, direct sequencing, PCR-RFLP, qPCR respectively, investigators found the presence of T790M mutation may be more extensive than we thought, even up to $60-80 \%$, fully illustrated that T790M mutation is the most common cause of EGFR-TKI resistance ${ }^{[4-6,13]}$. In addition Kuang, Y., et al discovered T790M genes can only be detected from 54\% patients whose plasma DNA were collected from CR patients in EGFR-TKI targeted therapy and there was no significant difference compared with tumor tissue sequencing data. However, examining the plasma of patients with known EGFR T790M mutation showed 71\% (5/7) of the patients whose plasma DNA was able to detect T790M EGFR, declaring EGFR T790M can be detected from plasma DNA of EGFR-TKI resistant patients and this non invasive method is also useful for monitoring drug resistance in the future, providing supplementary basis for guideline of subsequent drug use ${ }^{[14]}$. The ultimate aim of studying T790M mutation was to find an effective drug that can overcome TKI resistance associated with mutations. AZD9291 is the third generation oral drug and irreversible selective EGFR mutated inhibitor, which possessed strong anti-tumor efficacy and high selectivity for both EGFR-TKI sensitive mutations (EGFR+) and drug resistance mutations (T790M). The overall response rate and clinical benefit rate for T790M positive patients were $64 \%$ and $90 \%$, both of which were extremely higher than negative ones (23\% and 64\%), and PFS was also up to 13.5 months ${ }^{[15]}$. What's more, Rociletinib (CO-1686), as another third generation EGFR-TKI, whose overall response rate and clinical benefit rate reached to $64 \%$ and $90 \%$ respectively and PFS got up to 8-10 months was also achieved good therapeutic effect ${ }^{[16]}$. In conclusion, the third generation of EGFR-TKI will undoubtedly prove to be the essential substances to overcome drug resistance caused by T790M. Currently which is being studied for the third generation of EGFR-TKI includes HM61713, EGF816, ASP8273, besides domestic is also developing third generation EGFR-TKI, such as Avitinib and BPI-15086. According to the T790M mutation status, the current prevailing perception for NSCLC patients with EGFR sensitive mutation is, all the patients with resistance to the first generation or the second generation EGFR-TKI can be divided into two classes: Patients with second mutations of T790M will achieve good therapeutic effect adopted by the third generation of EGFR-TKI, however for the resistant patients with T790M negative status, clinical studies on the mechanisms of drug resistance may be the best choice ${ }^{[17]}$.

\section{Currently how to deal with NSCLC patients with resistance to the first generation of EGFR-TKI in clnical, especially before the third generation of EGFR-TKI listed?}

Clinical retrospective study of Faehling, M., et al found patients with previously effective erlotinib treatment who developed disease progression applying continuous EGFR-TKI therapy even can acquire an average survival time of 14.5 months or more, overall survival of nearly 50 months since the diagnosis. However patients who discontinued TKI treatment obtained an average survival time of only 2 months after progression, and overall survival of 28 months since the diagnosis, which was significantly lower than that of patients who continued to use TKI therapy after disease progression ${ }^{[18]}$. The Japanese scholar Hosomi Y., reported a multicenter, prospective observational study in ASCO 2014 on assessment of PD, Clinical practice treatment mode and curative effect of NSCLC patients with EGFR mutation positive receiving the first-line TKI therapy based on RECIST criteria and the observation showed PFS (R-PDFS) was 264 days while the clinical judgment of disease progression (C-PDFS) was almost 461 days. Patients developed without obvious clinical progression can acquire clinical stability for 6 months if performing continuous TKI treatment after disease progression. What's more, the prognosis of patients with initial TKI therapy appeared clinical progression was significantly better than that of patients with discontinued TKI treatment ${ }^{[19]} .2014$ ESMO results from ASPIRATION ,a prospective, randomized controlled study investigated by Park K et al suggested, continued erlotinib treatment after the RECIST PD can prolong PFS for 3.1 months (from 11.0 to 14.1 months) with no new adverse events ${ }^{[20]} .2015$ NCCN guidelines recommended of patients should apply erlotinib continual therapy when progress appeared in imaging diagnosis but no clinical symptoms were found. Retrospective investigation published by Nishie K, et al displayed NSCLC patients with EGFR sensitive mutation applied ongoing Gefitinib treatment followed with disease progression by TKI can acquire the median survival time for 32 months, whereas only 23 months were appeared among patients who received replacement for chemotherapy ${ }^{[21]}$. Therefore, from a point of view, for patients with initial effective treatment of TKI, disease progress in imaging diagnosis but no significant symptoms, both of disable TKI and replacement with chemotherapy is inferior to continual use for TKI to benefit patients.

Undoubtedly, it is necessary to use chemotherapy for patients with resistance, rapid progression of disease or obvious clinical symptoms, even cannot continue acquired benefit.

\section{Which is a better choice comparing chemotherapy alone and TKI combined with chemotherapy?}

A retrospective analysis on Comparing with the effects of single drug chemotherapy and erlotinib with chemotherapy combination in patients with acquired resistance which implanted by Goldberg SB, at Massachusetts General Hospital found Whatever adopting single drug chemotherapy or combined with platinum chemotherapy, even erlotinib with chemotherapy combination, the PFS are not superior than that of single chemotherapy ${ }^{[22]}$. 2014 ESMO Tony Mok reported the outcomes of IMPRESS study Which is a randomized, double-blinded, placebo-controlled phase III global multi center clinical trial, aimed to compare the efficacy and 
safety of ongoing Gefitinib associated with chemotherapy with single chemotherapy in advanced Gifitinib resistance NSCLC patients. Research results show that the two main endpoint was PFS was 5.4 months, Orr and DCR without significant difference, consequently it is not recommended adopting the second-line chemotherapy which contained two drugs based on platinum with Gefitinib combination for patients with disease progression ${ }^{[23]}$.

\section{How to choose the optimal therapy strategy at the end of the second line chemotherapy?}

Namba Y, in a retrospective research discovered the median survival time can reached 955 days for rechallenge TKI therapy; whereas only 615 days of median survival time can be reached if abandoning TKI rechallengement, suggesting the survival time was obviously higher for patients who received TKI rechallenge therapy than not $(p=0.0194)$. Nevertheless, T790M disappearance may occur in some part of patients undergoing chemotherapy, also with the second mutations of T790M, which may be explained as a new effective process for patients re-sensitive and re-challenged to TKI.

In addition, the survival time of patients who received multiple line therapies followed with TKI treatment was significantly longer than those who only received first-line or less line therapy ${ }^{[24]}$. Therefore it is necessary for patients to administrate with EGFR-TKI therapy as long as possible be under the premise of drug tolerance, however, if the patients cannot acquire evidential benefits of clinical symptoms, the second line chemotherapy should be performed, and TKI treatment should not be administrated until no more use of chemotherapy for patients. What's more, it is possible for NSCLC patients to be able to achieve benefits from TKI rechallenge therapy or multi line treatment at the end of the second line chemotherapy, and tumor resistance rebiopsy or participation in clinical research are also recommended for those who have failed in target therapy.

\section{How to perform the further cure facing the acquired resistance of the third generation TKI?}

Obviously patients with T790M (+) should be given the third generation TKI treatment ${ }^{[25]}$. A study published in the Nat Med 2015 discovered cell growth conditions using BA/F3 cells with L858R or T790M mutation which transected with C797S mutants or not in different concentrations of AZD9291 treatments. The data displayed the susceptibility of L858R or T790M mutated $\mathrm{Ba} / \mathrm{F} 3$ cells transfected with C797S mutant to AZD9291 was startling attenuated, which was also occurred in CO-1686,as the second generation of selective mutation EGFR-TKI targeted drug, exerting the good function to T790M positive NSCLC patients. In general, C797S mutation may be a mediator of AZD9291 acquired resistance in lung cancer patients, consequently, it is suggested that effective targeting therapy for C797S mutations need to be managed in patients with AZD9291 acquired resistance ${ }^{[26]}$. Nowadays, the new use of old drugs is also emerging. 2014 Clin Cancer Res reported that the combined treatment with metformin and TKI may reverse the efficacy of EGFR mutation NSCLC patients and prolong the overall survival time, which proposed a novel perspective for NSCLC target therapy ${ }^{[27]}$.

\section{Conclusion}

In summary, NSCLC patients undergoing EGFR-TKI acquired resistance are not a homogeneous population, but a collection of different types of patients which cannot be treated with the same method. We should deal with each case on its merits in clinical practice: The third generation of EGFR-TKI was the best choice for patients with T790M acquired resistance; applying the second biopsy under the condition permit to verify the specific mechanism of drug resistance; Distinguish various progresses for patients, such as slow, asymptomatic or local progression, and the continual EGFR-TKI (or combined with local therapy) therapy should not suspended until the patient can no longer acquired benefit from the TKI treatment. The effective second line therapy such as cisplatin or pemetrexed can relieve if symptomatic, rapid and extensive progress were appear, whereas if it is on the basis of combination chemotherapy, the application of EGFR-TKI should not be continued. Undoubtedly, which option on EGFR-TKI treatment undergo disease progress to take in the future is still needed to further distinguish the development pattern and the resistance mechanism, consequently adopting individualized therapy strategy.

\section{References}

1. Sharma, S.V., Bell, D.W., Settleman, J., et al. Epidermal growth factor receptor mutations in lung cancer. (2007) Nat Rev Cancer 7(3): 169-181. 2. Hata, A., Katakami, N., Yoshioka, H., et al. Rebiopsy of non-small cell lung cancer patients with acquired resistance to epidermal growth factor receptor-tyrosine kinase inhibitor: Comparison between T790M mutation-positive and mutation-negative populations. (2013) Cancer 119(24): 4325-4332.

3. Riely, G.J, Yu, H.A. EGFR: The Paradigm of an Oncogene-Driven Lung Cancer. (2015) Clin Cancer Res 21(10): 2221-2226.

4. Kuiper, J.L., Heideman, D.A., Thunnissen, E., et al. Incidence of T790M mutation in (sequential) rebiopsies in EGFR-mutated NSCLC-patients. (2014) Lung Cancer 85(1): 19-24.

5. Oxnard, G.R., Arcila, M.E., Sima, C.S., et al. Acquired resistance to EGFR tyrosine kinase inhibitors in EGFR-mutant lung cancer: distinct natural history of patients with tumors harboring the T790M mutation. (2011) Clin Cancer Res 17(6): 1616-1622.

6. Yu, H.A., Arcila, M.E., Rekhtman, N., et al. Analysis of tumor specimens at the time of acquired resistance to EGFR-TKI therapy in 155 patients with EGFR-mutant lung cancers. (2013) Clin Cancer Res 19(8): 2240-2247.

7. Uramoto, H., Yamada, T., Yano, S., et al. Prognostic value of acquired resistance-related molecules in Japanese patients with NSCLC treated with an EGFR-TKI. (2012) Anticancer Res 32(9): 3785-3790.

8. Zimmermann, S., Peters, S. Practical tips and tricks with recently approved molecular targeted agents in non-small-cell lung cancer. (2013) EJC 11(Suppl 2): 307-309. 
9. Rosell, R., Bivona, T.G., Karachaliou, N. Genetics and biomarkers in personalization of lung cancer treatment. (2013) Lancet 382(9893): 720731.

10. Yu, Z., Boggon, T.J., Kobayashi, S., et al. Resistance to an irreversible epidermal growth factor receptor (EGFR) inhibitor in EGFR-mutant lung cancer reveals novel treatment strategies. (2007) Cancer Res 67(21): 10417-10427.

11. Su, K.Y., Chen, H.Y., Li, K.C., et al. Pretreatment epidermal growth factor receptor (EGFR) T790M mutation predicts shorter EGFR tyrosine kinase inhibitor response duration in patients with non-small-cell lung cancer. (2012) J Clin Oncol 30(4): 433-440.

12. Yu, H.A., Arcila, M.E., Hellmann, M.D., et al. Poor response to erlotinib in patients with tumors containing baseline EGFR T790M mutations found by routine clinical molecular testing. (2014) Ann Oncol 25(2): 423-428.

13. Costa, C., Molina, M.A., Drozdowskyj, A., et al. The impact of EGFR T790M mutations and BIM mRNA expression on outcome in patients with EGFR-mutant NSCLC treated with erlotinib or chemotherapy in the randomized phase III EURTAC trial. (2014) Clin Cancer Res 20(7): 2001-2010.

14. Kuang, Y., Rogers, A., Yeap, B.Y., et al. Noninvasive detection of EGFR T790M in gefitinib or erlotinib resistant non-small cell lung cancer. (2009) Clin Cancer Res 15(8): 2630-2636.

15. Janne, P.A., Yang, J.C., Kim, D.W., et al. AZD9291 in EGFR inhibitor-resistant non-small-cell lung cancer. (2015) N Engl J Med 372(18): 1689-1699.

16. Sequist, L.V., Soria, J.C., Goldman, J.W., et al. Rociletinib in EGFR-mutated non-small-cell lung cancer. (2015) N Engl J Med 372(18): 17001709.

17. Steuer, C.E., Khuri, F.R., Ramalingam, S.S. The next generation of epidermal growth factor receptor tyrosine kinase inhibitors in the treatment of lung cancer. (2015) Cancer 121(8): 1-6.

18. Faehling, M., Eckert, R., Kamp, T., et al. EGFR-tyrosine kinase inhibitor treatment beyond progression in long-term Caucasian responders to erlotinib in advanced non-small cell lung cancer: a case-control study of overall survival. (2013) Lung Cancer 80(3): 306-312.

19. Yukio Hosomi, C.T., Kiyotaka Yoh, Yasushi Goto, et al. Observational study of treatment with epidermal growth factor receptor tyrosine kinase inhibitors (EGFR-TKI) in activating EGFR-mutation-positive (EGFRm+) advanced or recurrent non-small cell lung cancer (NSCLC) after radiologic progression to first-line therapy with EGFR-TKI. (2014) J Clin Oncol 2014 ASCO Annual Meeting Abstracts. 8071.

20. Park, K., Ahn, M., Yu, C., et al. ASPIRATION : First-line erlotinib (e) until and beyond recist progression (PD) in asian patients (pts) with EGFR mutation -positive (mut+) NSCLC. (2014) Annals of Oncology 2014 ESMO Abstract 12230 25(suppl 4): 426-427.

21. Nishie, K., Tomoya, K., Akihiro, T., et al. Epidermal growth factor receptor tyrosine kinase inhibitors beyond progressive disease: a retrospective analysis for Japanese patients with activating EGFR mutations. (2012) J Thorac Oncol 7(11): 1722-1727.

22. Goldberg, S.B., Oxnard, G.R., Digumarthy, S., et al. Chemotherapy with Erlotinib or chemotherapy alone in advanced non-small cell lung cancer with acquired resistance to EGFR tyrosine kinase inhibitors. (2013) Oncologist 18(11): 1214-1220.

23. Mok, T.S.K., Wu, Y., Nakagawa, K., et al. Gefitinib/Chemotherapy vs Chemotherapy in epidermal growth factor receptor (EGFR) mutation-positive non-small-cell lung cancer (NSCLC) after progression on first-line gefitinib: The phase III, randomised impress study. (2014) Annals of Oncology ESMO Abstract LBA2. 25(Suppl 4): 1-41.

24. Nishino, K., Imamura, F., Morita, S., et al. A retrospective analysis of 335 Japanese lung cancer patients who responded to initial gefitinib treatment. (2013) Lung Cancer 82(2): 299-304.

25. Mok, T., Yang, J.J., Lam, K.C., et al. Treating patients with EGFR-sensitizing mutations: first line or second line--is there a difference. (2013) J Clin Oncol 31(8): 1081-1088.

26. Thress, K.S., Paweletz, C.P., Felip, E., et al. Acquired EGFR C797S mutation mediates resistance to AZD9291 in non-small cell lung cancer harboring EGFR T790M. (2015) Nat Med 21(6): 560-562.

27. Li, L., Han, R., Xiao, H., et al. Metformin sensitizes EGFR-TKI-resistant human lung cancer cells in vitro and in vivo through inhibition of IL-6 signaling and EMT reversal. (2014) Clin Cancer Res 20(10): 2714-2726.

Ommega Online Publishers

Journal Title: International Journal of Cancer and Oncology (IJCO)

Journal Short Name: Int J Cancer Oncol
Journal ISSN: 2377-0902

E-mail: cancerandoncology@ommegaonline.com Website: www.ommegaonline.org 